\title{
Accessing the gluonic structure of light nuclei at a future electron-ion collider
}

\author{
Heikki Mäntysaari $\oplus^{1,2}$ and Björn Schenke ${ }^{3}$ \\ ${ }^{1}$ Department of Physics, University of Jyväskylä, P.O. Box 35, 40014 University of Jyväskylä, Finland \\ ${ }^{2}$ Helsinki Institute of Physics, P.O. Box 64, 00014 University of Helsinki, Finland \\ ${ }^{3}$ Physics Department, Brookhaven National Laboratory, Upton, New York 11973, USA
}

(Received 17 October 2019; published 13 January 2020)

\begin{abstract}
We show how exclusive vector meson production off light ions can be used to probe the spatial distribution of small- $x$ gluons in the deuteron and ${ }^{3} \mathrm{He}$ wave functions. In particular, we demonstrate how short-range repulsive nucleon-nucleon interactions affect the predicted coherent $J / \Psi$ production spectra. Fluctuations of the nucleon substructure are shown to have a significant effect on the incoherent cross section above $|t| \gtrsim 0.2 \mathrm{GeV}^{2}$. By explicitly performing the Jalilian-Marian-Iancu-McLerran-Weigert-Leonidov-Kovner (JIMWLK) evolution, we predict the $x$ dependence of coherent and incoherent cross sections in the electron-ion collider energy range. In addition to the increase of the average size of the nucleus with decreasing $x$, both the growth of the nucleons and subnucleonic hot spots are visible in the cross sections. The decreasing length scale of color charge fluctuations with decreasing $x$ is also present, but may not be observable for $|t|<1 \mathrm{GeV}^{2}$, if subnucleonic spatial fluctuations are present.
\end{abstract}

DOI: 10.1103/PhysRevC.101.015203

\section{INTRODUCTION}

Electron-proton and electron-nucleus collisions can be used to precisely probe the internal structure and dynamics of protons and nuclei. Deep inelastic scattering (DIS) measurements of electrons on protons, performed at HERA, in which the electron emits a virtual photon which scatters off the target proton, have provided a detailed picture of the internal quark and gluon structure of the proton [1,2]. These measurements have revealed that at high energies (small longitudinal momentum fraction $x$ ), the proton structure is dominated by gluons.

Recently, the authors argued that the spatial distribution of small- $x$ gluons in the proton fluctuates event by event. This is evident from studying exclusive vector meson production. In coherent scattering where the target proton remains intact, the average shape of the proton is probed. In incoherent diffraction where the target dissociates, on the other hand, one is sensitive to the amount of event-by-event fluctuations $[3,4]$ (see also Refs. [5-9]). These nucleon shape fluctuations have also been suggested to have a measurable effect in heavy nuclei [10]. In addition to DIS experiments, the nucleon shape can be studied in hadronic collisions. In particular, the proton-lead collisions at the Large Hadron Collider (LHC) have revealed unexpected collective phenomena (for a review, see, e.g., Ref. [11]). One potential source of the observed collectivity is the final state response to the initial state geometry. To verify

Published by the American Physical Society under the terms of the Creative Commons Attribution 4.0 International license. Further distribution of this work must maintain attribution to the author(s) and the published article's title, journal citation, and DOI. Funded by $S C O A P^{3}$. this interpretation and to disentangle it from other sources of correlations, a good understanding of the proton geometry (and nucleon geometry in the nucleus) is required. It was shown that the flow measurements in LHC proton-lead collisions are compatible with a hydrodynamically evolving quark gluon plasma (QGP), initiated with a color glass condensate (CGC) initial condition, only if proton geometry fluctuations are included $[12,13]$. Similarly, the geometry fluctuations in the nucleons were found to be important in a global analysis of lead-lead and proton-lead flow data [14]. Also, analysis of the elastic proton-proton differential cross section, measured by the TOTEM Collaboration [15] at high energy, has revealed indications for a hot spot structure of the proton [16].

The structure of light nuclei, such as the deuteron and helium at large $x$, is well known from low-energy scattering experiments. However, little is known about the spatial distribution of small- $x$ gluons in these systems. To access the fundamental information on the small- $x$ gluon structure in light nuclei, as well as to provide input to models aimed at describing deuteron-gold and helium-gold collisions at the Relativistic Heavy Ion Collider (RHIC) [17-19], one needs new measurements possible at a future electron-ion collider (EIC) [20].

In this work we study the EIC's potential to constrain the spatial distribution of small- $x$ gluons in light nuclei via measurements of exclusive vector meson production. In particular, we compute differential coherent and incoherent cross sections for $J / \Psi$ production within both the IPsat model [21] and the color glass condensate framework, which includes the explicit solution of the Jalilian-Marian-IancuMcLerran-Weigert-Leonidov-Kovner (JIMWLK) [22-25] evolution equations.

This article is structured as follows. In Sec. II we discuss how the deuteron and helium structure is obtained in terms of 
the nucleon constituents. In Sec. III it is shown how diffractive scattering processes are calculated at high energy in the dipole picture. The required dipole-nucleus scattering amplitudes encoding the target structure are obtained as discussed in Sec. IV. The resulting energy evolution for the structure of light nuclei is illustrated in Sec. V, and predictions for the future electron-ion collider are shown in Sec. VI. Our conclusions and outlook are presented in Sec. VII.

\section{STRUCTURE OF LIGHT IONS}

The density profiles of light nuclei can be calculated rather accurately using theoretical methods to describe strongly correlated quantum systems. We extract the nucleon positions in light $(A=2,3)$ nuclei from such calculations configuration by configuration. In the high-energy scattering processes at the EIC one is sensitive to the small- $x$ gluon distribution, about which we have to make additional assumptions given the distribution of nucleons. Future experimental data in combination with our calculations will be able to better constrain the small- $x$ structure of light nuclei.

To quantify the uncertainty in the current understanding of the deuteron structure, and to study the capabilities of the EIC for constraining the model uncertainties, we apply two different deuteron wave functions in this work. First, we use the deuteron wave function from Refs. [26,27], obtained using the Argonne v18 (AV18) two-nucleon potential, including both $S$ and $P$ wave contributions, referred to as Argonne in this paper. This $a b$ initio calculation includes both attractive and repulsive nucleon-nucleon interactions.

For comparison, we also employ the commonly used Hulthen wave function [28] in which the distribution of the proton-neutron separation $d_{\mathrm{pn}}$ is parametrized as

$$
\phi_{\mathrm{pn}}\left(d_{\mathrm{pn}}\right)=\frac{1}{\sqrt{2 \pi}} \frac{\sqrt{a b(a+b)}}{b-a} \frac{e^{-a d_{\mathrm{pn}}}-e^{-b d_{\mathrm{pn}}}}{d_{\mathrm{pn}}} .
$$

The experimentally determined parameters used in this work are $a=0.228 \mathrm{fm}^{-1}$ and $b=1.18 \mathrm{fm}^{-1}$ [28]. Note that these parameters are fixed by low-energy data, and there is no a priori reason why the small- $x$ gluonic distribution should resemble this precisely.

The distribution of the proton-neutron separation $d_{\mathrm{pn}}$ (in three dimensions) obtained from these two parametrizations is shown in Fig. 1. Both the Hulthen and Argonne potential wave functions produce deuterons with roughly the same root mean square size ( $3.93 \mathrm{fm}$ in the case of the Argonne potential, 4.07 $\mathrm{fm}$ in the case of Hulthen). The largest difference between the wave functions is that the repulsive short-range nucleonnucleon interactions suppress small proton-neutron separations in the Argonne wave function compared to the Hulthen case. We note that recently the short-range correlations in deuterons (and other light nuclei) were studied in detail, in connection with the EMC effect (see, e.g., Refs. [29-31]).

The nucleonic structure of ${ }^{3} \mathrm{He}$ is obtained from the Monte Carlo calculation with AV18+UIX interaction [32]. AV18 refers to the same two-nucleon potential used to obtain the deuteron configurations discussed above. In practice, we use the same database of configurations that is used in Ref. [33] and available in the PHOBOS Monte Carlo Glauber imple-

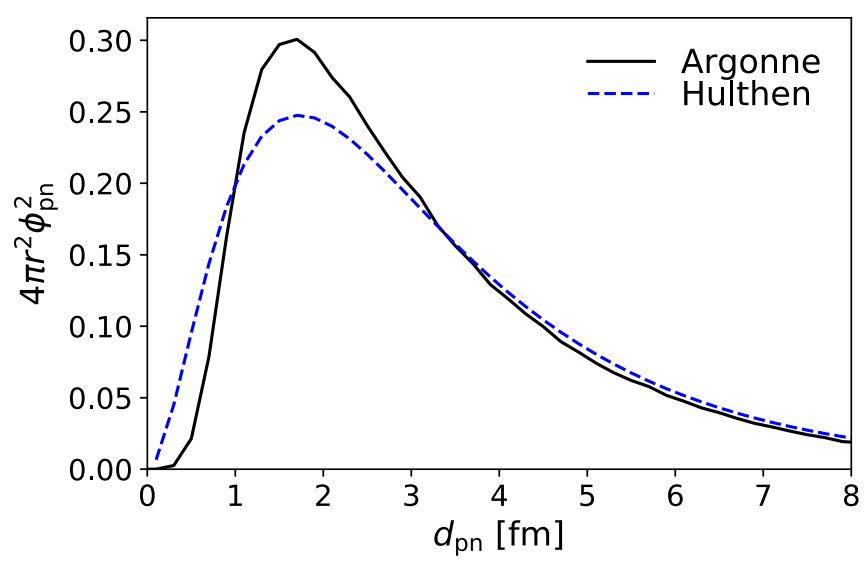

FIG. 1. Deuteron size distribution from the Hulthen and Argonne potential wave functions.

mentation [34]. These configurations reproduce the charge radii and form factors of helium-3, as well as the relative separation of proton pairs.

\section{DIFFRACTIVE DEEP INELASTIC SCATTERING}

Diffractive vector meson production is a powerful probe of the spatial structure of nuclei, as the total momentum transfer, which is the Fourier conjugate to the impact parameter, is measurable. These processes are divided in two categories: in coherent scattering the target hadron remains in its ground state, and in incoherent diffraction the target dissociates. In either case there is no net color charge transferred to the probe, which leads to an experimentally observable rapidity gap between the produced vector meson and the target or target remnants.

In the Good-Walker picture [35], the coherent cross section is obtained by calculating the average scattering amplitude for the Fock states of the probing virtual photon that diagonalize the scattering matrix. At high energy, these states are quarkantiquark states with fixed transverse separation $\mathbf{r}$ scattering off a fixed target configuration. The coherent cross section can be written as $[5,36]$

$$
\frac{d \sigma^{\gamma^{*} N \rightarrow V N}}{d t}=\frac{1}{16 \pi}\left|\left\langle\mathcal{A}^{\gamma^{*} N \rightarrow V N}\left(x_{\mathbb{P}}, Q^{2}, \boldsymbol{\Delta}\right)\right\rangle\right|^{2},
$$

where the average \langle\rangle refers to the average over all possible target configurations, and $N$ can be a proton or a nucleus.

The scattering amplitude $\mathcal{A}^{\gamma^{*} N \rightarrow V N}\left(x_{\mathbb{P}}, Q^{2}, \boldsymbol{\Delta}\right)$ can be written as a Fourier transform from coordinate space to momentum space [36],

$$
\begin{aligned}
& \mathcal{A}_{T, L}^{\gamma^{*} N \rightarrow V N}\left(x_{\mathbb{P}}, Q^{2}, \boldsymbol{\Delta}\right) \\
& =i \int d^{2} \mathbf{r} \int d^{2} \mathbf{b} \int \frac{d z}{4 \pi} \\
& \quad \times\left(\Psi^{*} \Psi_{V}\right)_{T, L}\left(Q^{2}, \mathbf{r}, z\right) e^{-i[\mathbf{b}-(1-z) \mathbf{r}] \cdot \Delta} 2 N\left(\mathbf{b}, \mathbf{r}, x_{\mathbb{P}}\right) .
\end{aligned}
$$

Here, the two-dimensional vector $\Delta$ is the transverse momentum transfer to the target, with $|\boldsymbol{\Delta}| \approx \sqrt{-t}$. The transverse momentum transfer $\boldsymbol{\Delta}$ is actually the Fourier conjugate to $\mathbf{b}-(1-z) \mathbf{r}$ because of the contribution from the nonforward 
vector meson wave function [36,37]. The impact parameter b points to the center of the dipole from the center of the target. The virtual photon to quark-antiquark dipole splitting is described by the virtual photon wave function $\Psi$, and the formation of a vector meson is encoded in the vector meson wave function $\Psi_{V}$. In this work we use the boosted Gaussian wave function parametrization from Ref. [36] (see also Ref. [38]). Note that the limited knowledge of the vector meson wave function leads to significant uncertainties in the absolute normalization of the cross section (up to $\sim 50 \%$; see, e.g., Ref. [39]). The interaction of a dipole with transverse size $\mathbf{r}$ and impact parameter $\mathbf{b}$ with the target (proton or nucleus) is described in terms of the dipole amplitude $N\left(\mathbf{r}, \mathbf{b}, x_{\mathbb{P}}\right)$. The target structure is probed at Bjorken- $x$

$$
x_{\mathbb{P}}=\frac{Q^{2}+M_{V}^{2}-t}{Q^{2}+W^{2}-m_{N}^{2}},
$$

which can be interpreted as the longitudinal momentum fraction of the proton carried by the color-neutral "pomeron" exchanged with the diffractively produced vector meson $(J / \Psi$ in this work). Here $W$ is the center-of-mass energy in the photon-nucleon scattering and $m_{N}$ the nucleon mass.

If we calculate the total cross section for diffractive vector meson production and subtract the coherent contribution, we obtain the incoherent diffractive cross section. Following Ref. [8] (see also Refs. [5-7,9]), the incoherent cross section becomes

$$
\begin{aligned}
\frac{d \sigma^{\gamma^{*} N \rightarrow V N^{*}}}{d t}= & \frac{1}{16 \pi}\left(\left\langle\left|\mathcal{A}^{\gamma^{*} N \rightarrow V N}\left(x_{\mathbb{P}}, Q^{2}, \boldsymbol{\Delta}\right)\right|^{2}\right\rangle\right. \\
& \left.-\left|\left\langle\mathcal{A}^{\gamma^{*} N \rightarrow V N}\left(x_{\mathbb{P}}, Q^{2}, \boldsymbol{\Delta}\right)\right\rangle\right|^{2}\right) .
\end{aligned}
$$

As the incoherent cross section is a variance, it measures the amount of fluctuations in the diffractive scattering amplitude. Additionally, the coherent cross section depends on the average scattering amplitude and consequently on the average target structure. These two cross sections then make it possible to constrain the event-by-event fluctuating structure of the hadron, as shown in the case of protons in Ref. [3] and with heavy nuclei in Ref. [10].

There are two phenomenological corrections to the results presented above (see Ref. [36]). First, in the dilute limit where two gluons are exchanged, one actually probes a two-gluon distribution of the target. A dominant contribution to the cross section then originates from the configuration where one of the gluons is very soft, and in this limit one can relate the result to the standard collinear factorization gluon distribution function by introducing the skewedness correction [40]. However, applicability of this correction in the saturation region is not clear. The second correction originates from the fact that usually one assumes the dipole scattering amplitude to be purely real. Both of these contributions mostly affect the overall normalization of the cross section (the $t$ and energy dependencies are weak; see, e.g., Ref. [10]). As the overall normalization has a large uncertainty originating from the poorly constrained vector meson wave function, we only add these corrections approximatively. The skewedness correction is estimated as a $40 \%$ increase to the cross section. A similar $10 \%$ real part correction is applied to the results obtained using the IPsat parametrization where the dipole amplitude is purely real.

\section{DIPOLE-TARGET SCATTERING}

We consider two different descriptions for the dipoletarget interaction that allow us to obtain the dipole amplitude $N\left(\mathbf{r}, \mathbf{b}, x_{\mathbb{P}}\right)$. These are the IPsat parametrization, in which the geometry does not evolve in $x$ and the Bjorken- $x$ dependence of the saturation scale $Q_{\mathrm{s}}^{2}$ (or density) is parametrized by fitting to HERA data. This simple parametrization is compared with an explicit color glass condensate framework calculation in which the energy evolution of the Wilson lines (and consequently the target geometry) is obtained by solving perturbative evolution equations. Summaries of these two approaches are presented below.

\section{A. IPsat model}

In the IPsat parametrization the saturation scale $Q_{\mathrm{s}}^{2}$ depends on the impact parameter, and the dipole-proton scattering amplitude is written as

$$
N^{p}(\mathbf{r}, \mathbf{b}, x)=1-\exp \left[-\mathbf{r}^{2} F(x, \mathbf{r}) T_{p}(\mathbf{b})\right],
$$

where the transverse density profile function is assumed to be Gaussian: $T_{p}(\mathbf{b})=\frac{1}{2 \pi B_{p}} e^{-\mathbf{b}^{2} /\left(2 B_{p}\right)}$. The function $F$ contains the Dokshitzer-Gribov-Lipatov-Altarelli-Parisi (DGLAP) evolved gluon distribution $x g$ :

$$
F\left(x, \mathbf{r}^{2}\right)=\frac{\pi^{2}}{2 N_{\mathrm{c}}} \alpha_{\mathrm{s}}\left(\mu_{0}^{2}+\frac{C}{r^{2}}\right) x g\left(x, \mu_{0}^{2}+\frac{C}{r^{2}}\right) .
$$

The free parameters of the model $\left(\mu_{0}^{2}, C, B_{p}\right.$, and the initial condition for the DGLAP evolution of $x g$ ) are fixed by fitting the HERA data in Ref. [41] (see also Ref. [42]).

In Refs. [3,4], this parametrization was generalized to the case where the proton has a fluctuating substructure consisting of $N_{q}$ "hot spots" by replacing

$$
T_{p}(\mathbf{b}) \rightarrow \frac{1}{N_{q}} \sum_{i=1}^{N_{q}} T_{q}\left(\mathbf{b}-\mathbf{b}_{i}\right)
$$

with

$$
T_{q}(\mathbf{b})=\frac{1}{2 \pi B_{q}} e^{-\mathbf{b}^{2} /\left(2 B_{q}\right)} .
$$

Here $B_{q}$ is the Gaussian width of each hot spot. The locations of the hot spots are sampled from a Gaussian distribution with width $B_{q c}$. These parameters are constrained as in Ref. [4] by requiring a simultaneous description of the coherent and incoherent $J / \Psi$ photoproduction data from HERA [43] at $W=75 \mathrm{GeV}$. Unlike in Ref. [4], the sampled hot spot locations are shifted to keep the center of mass of the nucleon at the origin. This keeps the deuteron and helium sizes unchanged when the fluctuations are included, but effectively makes the nucleons smaller and consequently the used parameters deviate slightly from the ones used in Ref. [4]. In the case of the IPsat parametrization, we use parameters $B_{q c}=$ $4.5 \mathrm{GeV}^{-2}$ and $B_{q}=1.0 \mathrm{GeV}^{-2}$. 
The dipole-proton scattering amplitude $N$ discussed above can be generalized to the dipole-nucleus case as

$$
N^{A}(\mathbf{r}, \mathbf{b}, x)=1-\prod_{i=1}^{A}\left[1-N^{p}\left(\mathbf{r}, \mathbf{b}-\mathbf{b}_{i}, x_{\mathbb{P}}\right)\right],
$$

where $\mathbf{b}_{i}$ are the transverse positions of the nucleons, sampled from the nucleus wave function discussed in Sec. II. Within our framework, this procedure is equivalent to summing the density profiles of the nucleons to obtain that of the nucleus.

Several pieces of data prefer the presence of additional fluctuations of the normalization of $Q_{s}^{2}[3,4,44,45]$. We thus allow the (squared) saturation scale $Q_{s}^{2}$ of the individual hot spots to fluctuate around its expectation value $\left\langle Q_{s}^{2}\right\rangle$ following a log-normal distribution:

$$
P\left(\ln \left(Q_{s}^{2} /\left\langle Q_{s}^{2}\right\rangle\right)\right)=\frac{1}{\sqrt{2 \pi} \sigma} \exp \left[-\frac{\ln ^{2}\left(Q_{s}^{2} /\left\langle Q_{s}^{2}\right\rangle\right)}{2 \sigma^{2}}\right] .
$$

Here we use $\sigma=0.65$ adjusted to get a better description of the small- $|t|$ part of the incoherent $J / \Psi$ photoproduction off protons as measured by HERA. (In Refs. [3,4] with the IPsat parametrization where we did not shift center of mass to origin, we used the comparable value $\sigma=0.5$ determined in Ref. [45] based on observed multiplicity fluctuations in proton-proton collisions.) The sampled saturation scales are then rescaled to keep the average $\left\langle Q_{s}^{2}\right\rangle$ unchanged, as the expectation value of the log-normal distribution is not $\left\langle Q_{s}^{2}\right\rangle$ (see the discussion in Ref. [4]).

\section{B. Color glass condensate}

The eikonal propagation of a quark through the color field of the target at transverse coordinate $\mathbf{x}$ is determined by the Wilson lines $V(\mathbf{x})$, which describe the color rotation of the quark state. The Wilson lines at each point in the transverse plane are obtained from the color glass condensate effective theory calculation [44,46-48]. The details of the computation, summarized below, are exactly the same as in Refs. [3,4].

The target's color charge densities $\rho^{a}(\mathbf{x})$ in the transverse plane are assumed to be random, and sampled from the McLerran-Venugopalan (MV) model [49], where $\left\langle\rho^{a}\left(x^{-}, \mathbf{x}\right)\right\rangle=0$, and

$$
\left\langle\rho^{a}\left(x^{-}, \mathbf{x}\right) \rho^{b}\left(y^{-}, \mathbf{y}\right)\right\rangle=\delta^{a b} \delta^{(2)}(\mathbf{x}-\mathbf{y}) \delta\left(x^{-}-y^{-}\right) g^{2} \mu^{2},
$$

where $a$ and $b$ are color indices. The color charge density $g \mu$ is proportional to the saturation scale $Q_{s}\left(x_{\mathbb{P}}, \mathbf{x}, \mathbf{b}\right)$ as $Q_{s}=c g^{2} \mu$, with $c=0.7$ when nucleon shape fluctuations are included and $c=0.65$ without, as in Ref. [4] (see also Ref. [50]). The saturation scale $Q_{s}^{2}$, which is a proxy for the nucleon density, is obtained from the IPsat parametrization of the dipole amplitude, presented previously. It is defined via the relation

$$
N\left(\mathbf{r}^{2}=2 / Q_{s}^{2}, \mathbf{b}, x\right)=1-e^{-1 / 2} .
$$

After the saturation scale at every point in the transverse plane, $Q_{\mathrm{s}}^{2}(\mathbf{b})$, is obtained, we can sample color charges according to Eq. (12) and solve the Yang-Mills equations to determine the Wilson lines at every transverse position [48]:

$$
V(\mathbf{x})=P \exp \left(-i g \int d x^{-} \frac{\rho\left(x^{-}, \mathbf{x}\right)}{\nabla^{2}+\tilde{m}^{2}}\right) .
$$

Here $P$ indicates path ordering. To suppress long-distance Coulomb tails, an effective mass regulator $\tilde{m}$ is introduced. In general one expects $\tilde{m} \sim \Lambda_{\mathrm{QCD}}$, and here we use $\tilde{m}=$ $0.4 \mathrm{GeV}$ as constrained in Ref. [4] by comparing with the HERA $J / \Psi$ photoproduction data.

All nucleon shape and density fluctuations are included via $Q_{s}^{2}(\mathbf{b})$ calculated from the IPsat model, where geometry fluctuations are included as discussed in Sec. IV A. Here we use the parameters $B_{q c}=4.0 \mathrm{GeV}^{-2}$ and $B_{q}=0.3 \mathrm{GeV}^{-2}$, and $\sigma=0.5$ to determine the magnitude of the $Q_{s}^{2}$ fluctuations, as in Ref. [3].

After the Wilson lines at the initial $x_{0}=0.01$ are sampled as discussed above, the evolution towards smaller $x$ is obtained by solving the JIMWLK renormalization group equation [22-25]. Here we use exactly the same setup as in Ref. [51], which we summarize below.

The JIMWLK equation describes the rapidity evolution of a Wilson line and can be written as a stochastic Langevin equation [52]

$$
\frac{d}{d y} V(\mathbf{x})=V(\mathbf{x})\left(i t^{a}\right)\left[\int d^{2} \mathbf{z} \varepsilon_{\mathbf{x}, \mathbf{z}}^{a b, i} \xi_{\mathbf{z}}(y)_{i}^{b}+\sigma_{\mathbf{x}}^{a}\right],
$$

where $t^{a}$ is an $\mathrm{SU}(3)$ generator in the fundamental representation. The evolution (15) can be seen as a random walk in the color space, where the random noise $\xi$ is a local Gaussian variable with variance

$$
\left\langle\xi_{\mathbf{x}, i}^{a}(y) \xi_{\mathbf{y}, j}^{b}\left(y^{\prime}\right)\right\rangle=\delta^{a b} \delta^{i j} \delta_{\mathbf{x y}}^{(2)} \delta\left(y-y^{\prime}\right) .
$$

The coefficient of the noise is

$$
\varepsilon_{\mathbf{x}, \mathbf{z}}^{a b, i}=\left(\frac{\alpha_{\mathrm{s}}}{\pi}\right)^{1 / 2} K_{\mathbf{x}-\mathbf{z}}^{i}\left[1-V_{\mathbf{x}}^{\dagger} V_{\mathbf{z}}\right]^{a b},
$$

and the kernel reads

$$
K_{\mathbf{x}}^{i}=\frac{x^{i}}{\mathbf{x}^{2}}
$$

The deterministic drift term $\sigma_{\mathbf{x}}^{a}$ can be eliminated from the equation following Ref. [53], which avoids the requirement to evaluate Wilson lines in the adjoint representation [as appearing in Eq. (17)] and makes the numerical solution more efficient.

The JIMWLK kernel $K_{\mathbf{x}}^{i}$ has a powerlike structure, and consequently the evolution generates long-distance Coulomb tails [similar to those regulated at the initial condition by the mass parameter $\tilde{m}$ in Eq. (19)]. This would result in an exponentially growing cross section with rapidity $[54,55]$ and violate the Froissart bound [56]. To avoid this we follow Ref. [55] and introduce effective confinement scale effects by using a modified kernel,

$$
K_{\mathbf{x}}^{i} \rightarrow m|\mathbf{x}| K_{1}(m|\mathbf{x}|) \frac{x^{i}}{\mathbf{x}^{2}} .
$$

Here the modified Bessel function $K_{1}$ suppresses contributions at distance scale $\gtrsim 1 / m$. In this work we use $m=0.2 \mathrm{GeV}$ as constrained in Ref. [51] to be compatible with the HERA 

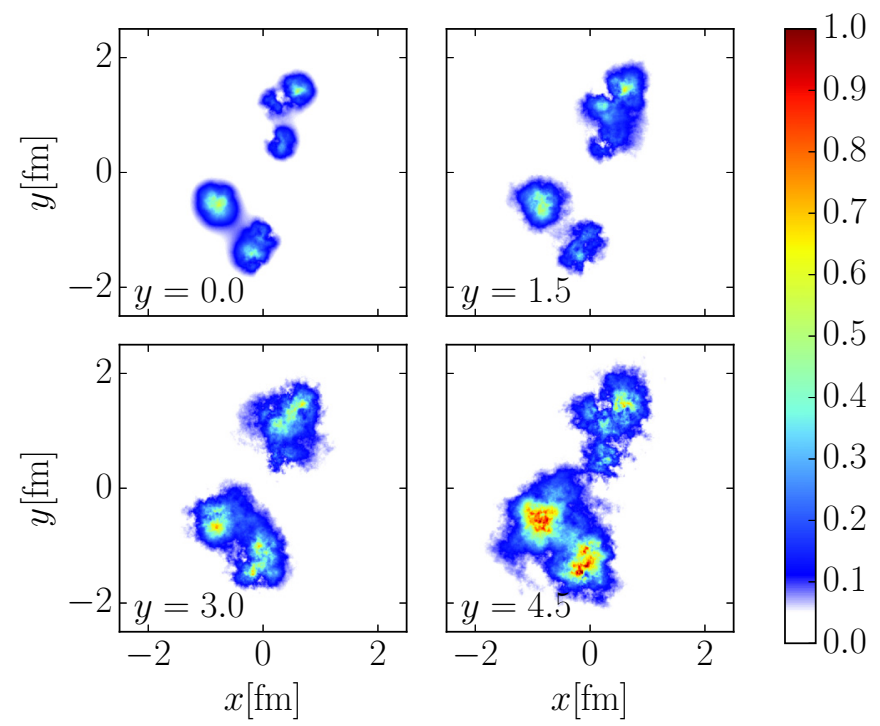

FIG. 2. Illustration of the deuteron density profile and its evolution in the case where nucleon shape fluctuations are included. The density is represented by $1-\operatorname{Re} \operatorname{Tr} V / N_{\mathrm{c}}$.

structure function data when the evolution is performed at fixed coupling $\alpha_{\mathrm{s}}=0.21$. In Ref. [51] we showed that the diffractive cross sections are sensitive to the infrared regulator $m$ only at small $t$ (if the strong-coupling constant is adjusted to keep the evolution speed compatible with the structure function data).

Calculations are performed on a two-dimensional lattice with transverse spacing $a=0.01 \mathrm{fm}$ and $L=13 \mathrm{fm}$ in the case of deuterons and $L=10 \mathrm{fm}$ with helium (note that larger lattices are needed with deuterons to accurately describe the tail of the distribution shown in Fig. 1). We checked that smaller lattice spacings do not alter the results. For more details on the JIMWLK evolution and its implementation on a lattice, the reader is referred to Ref. [51].

\section{ENERGY EVOLUTION OF LIGHT NUCLEI}

In this section we consider the CGC framework of Sec. IV B and illustrate the evolution of individual deuteron and ${ }^{3} \mathrm{He}$ configurations. Results for the deuteron including proton and neutron structure fluctuations and evolution over a few units of rapidity (note that $x=x_{0} e^{-y}$ with $x_{0}=0.01$ in this work) are shown in Fig. 2. The deuteron density is characterized by the trace of the Wilson line $1-\operatorname{Re} \operatorname{Tr} V / N_{\mathrm{c}}$. The evolution first washes out the proton substructure (as already noted in Ref. [55]), and eventually the nucleons grow enough to create one large region of gluon matter. However, with a typical proton-neutron transverse separation of $\sim 2 \mathrm{fm}$ one needs to go to very low $x$ in order to see the two nucleons merging. Similarly, in case of ${ }^{3} \mathrm{He}$, evolution for one example configuration (with nucleon substructure) is shown in Fig. 3, where very similar effects can be seen. To quantify how the presence of two nucleons affects the deuteron evolution in comparison to the single nucleon case, we study the deuteron density evolution as a function of rapidity, $y=\ln \frac{x_{0}}{x}$. In this case we fix the deuteron transverse size to a typical value
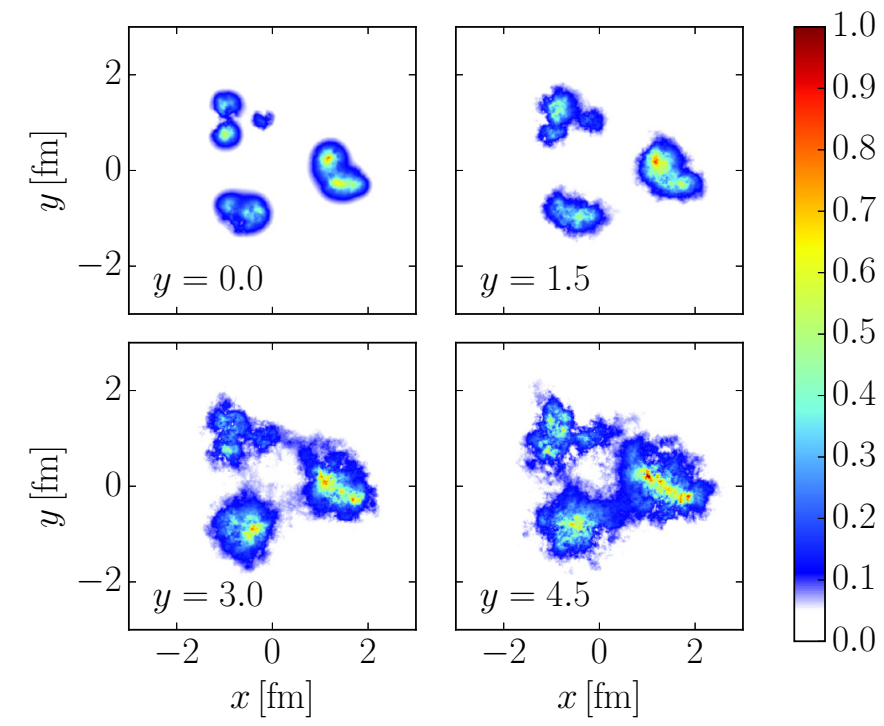

FIG. 3. Illustration of the helium-3 density evolution with nucleon shape fluctuations. The density is represented by $1-$ $\operatorname{Re} \operatorname{Tr} V / N_{\mathrm{c}}$.

$d_{\mathrm{pn}}=1.5 \mathrm{fm}$ and quantify the density by calculating the average dipole-deuteron scattering amplitude with a fixed size dipole with $|\mathbf{r}|=0.2 \mathrm{fm}$. Note that this is a typical scale for the dipoles contributing to the $J / \Psi$ production [36].

For comparison, we show the case where we consider the deuteron to consist of two independently evolved nucleons. In that case, the scattering amplitude $N_{\mathrm{pn}}$ for the proton-neutron system at point $b$ (where $b=0$ is the center of the deuteron) is obtained following Eq. (10):

$$
\begin{aligned}
N_{\mathrm{pn}}(r, b)= & N\left(r,\left|b-d_{\mathrm{pn}} / 2\right|\right)+N\left(r,\left|b+d_{\mathrm{pn}} / 2\right|\right) \\
& -N\left(r,\left|b-d_{\mathrm{pn}} / 2\right|\right) N\left(r,\left|b+d_{\mathrm{pn}} / 2\right|\right),
\end{aligned}
$$

where $N=N^{p}$ is the dipole-nucleon scattering amplitude.

The impact parameter dependence of the dipole amplitude is shown in Fig. 4, where the solid lines refer to the deuteron

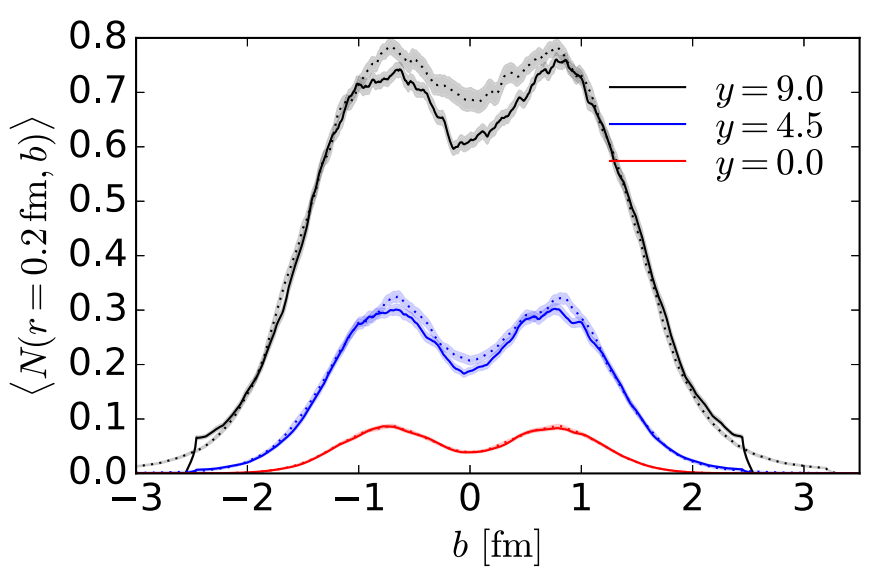

FIG. 4. Deuteron density measured by the fixed size dipole as a function of the impact parameter. The proton-neutron separation is $d_{\mathrm{pn}}=1.5 \mathrm{fm}$. The dashed line shows the result in the case where the proton and neutron are evolved independently. 


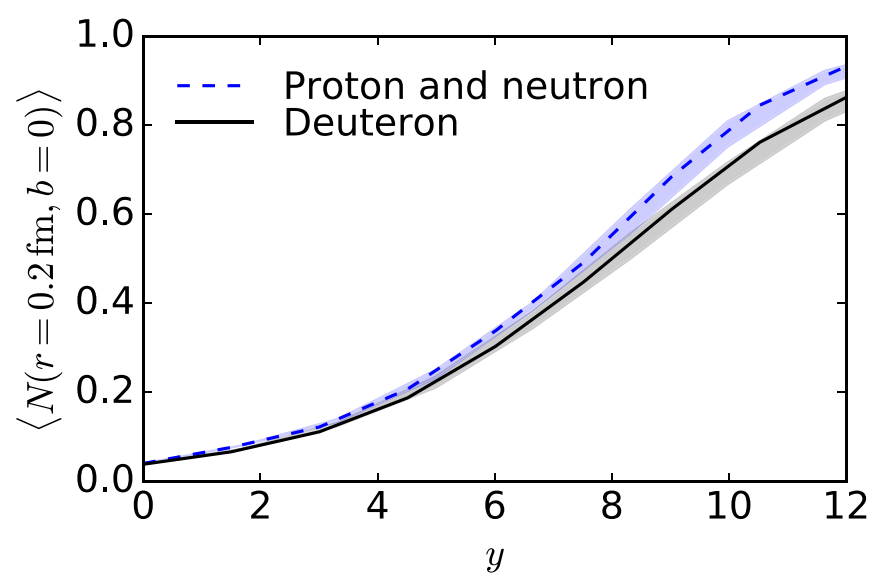

FIG. 5. Evolution of the deuteron density at the origin for a proton-neutron separation of $d_{\mathrm{pn}}=1.5 \mathrm{fm}$ (solid line), compared to the case where the two nucleons evolve independently (dashed line). The density is measured as the average interaction strength with the fixed size dipole. The lines are our standard choice with infrared regulator $m=0.2 \mathrm{GeV}$, and the band reflects the variation when $m$ is changed from $0.1 \mathrm{GeV}$ (lower) to $0.4 \mathrm{GeV}$ (upper).

and dotted lines assume independently evolving nucleons. We find that at the beginning of the evolution the two nucleons are separated enough spatially and the deuteron evolution is very close to the independent nucleon case. Only at large rapidities $y \gtrsim 4$ do we start to observe small deviations. We note that the rapidity at which this deviation begins depends heavily on the deuteron size $d_{\mathrm{pn}}$, and for smaller deuterons enhanced nonlinear effects in the dense region start to slow down the evolution earlier.

The rapidity dependence of the dipole amplitude at zero impact parameter is shown in Fig. 5. The evolution is identical in both cases until the proton and neutron grow so much that they start to overlap significantly at $y \gtrsim 5$, when the nonlinear effects start to decrease the evolution speed slightly compared with the independent nucleon case.

To demonstrate that our analysis is insensitive to the infrared regulator $m$, we vary $m$ in the JIMWLK kernel (19) and extract the same dipole amplitude evolution at zero impact parameter. The value of the strong-coupling constant for each infrared regulator is constrained in Ref. [51] from fits to HERA data. In addition to our standard choice $m=0.2 \mathrm{GeV}$ and $\alpha_{\mathrm{s}}=0.21$, we use $m=0.1 \mathrm{GeV}$ with $\alpha_{\mathrm{s}}=0.19$ and $m=$ $0.3 \mathrm{GeV}$ with $\alpha_{\mathrm{s}}=0.225$. As can be seen from Fig. 5, the evolution is very similar in all three cases. This was not entirely obvious, because the scenario of smaller $m$ and smaller $\alpha_{\mathrm{s}}$, for example, leads to faster evolution of the low-momentum modes, which dominate the evolution of the nucleon size. For larger dipoles the sensitivity to infrared regulators should be enhanced, but contributions from large dipoles to vector meson production is suppressed exponentially.

\section{PREDICTIONS FOR THE FUTURE ELECTRON-ION COLLIDER}

We consider $J / \Psi$ production in the kinematical range accessible with a future electron-ion collider, where the center-

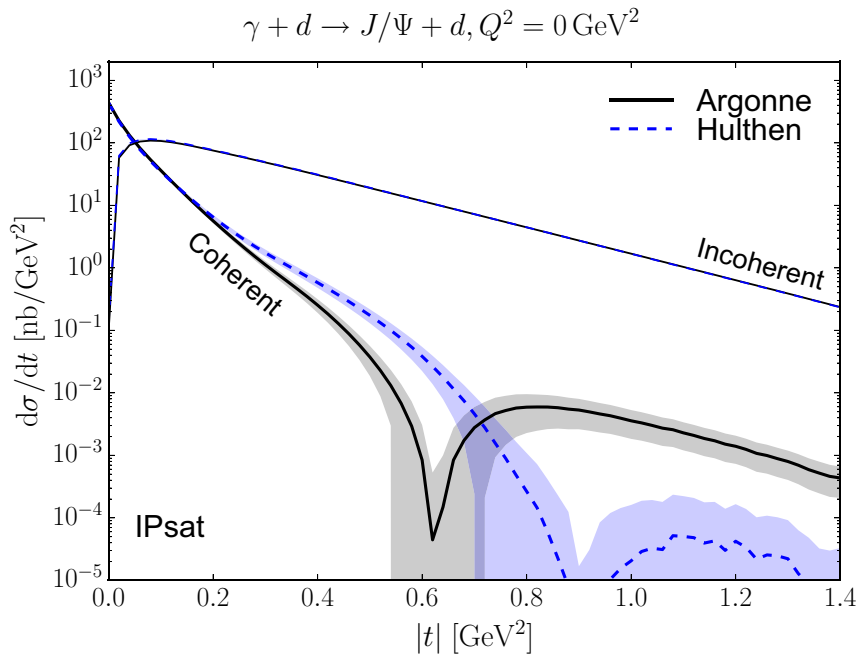

FIG. 6. Coherent and incoherent $J / \Psi$ production cross sections at $x_{\mathbb{P}}=0.01$ as a function of $t$, with two different wave functions to describe the deuteron structure. Nucleon shape fluctuations are not included.

of-mass energies can reach up to $\sqrt{s_{N N}}=140 \sqrt{Z / A} \mathrm{GeV}$ [57]. This allows the reach of $x_{\mathbb{P}}$ values down to $x_{\mathbb{P}} \sim$ $10^{-4}-10^{-3}$ in $J / \Psi$ photoproduction in electron-deuteron and electron-helium collisions.

\section{A. Short-range nucleon-nucleon correlations in the deuteron}

The structure of light nuclei at low energy scales is well known, but so far it has not been possible to probe the distribution of small- $x$ gluons in, e.g., the deuteron wave function. To demonstrate that the future electron-ion collider can do detailed imaging of the small- $x$ gluon distributions, we first study vector meson production off deuterons using two different realistic wave functions to describe the protonneutron separation in the deuteron, assuming that small- $x$ gluons are distributed around the nucleons.

The difference between the used Hulthen and Argonne wave functions is that the short-range nucleon-nucleon correlations that suppress configurations where proton and neutron are close to each other $\left(d_{\mathrm{pn}} \lesssim 0.5 \mathrm{fm}\right)$ are included in the Argonne wave function (see Fig. 1). For simplicity the nucleon shape fluctuations are not included in this analysis.

The obtained $J / \Psi$ photoproduction cross sections for coherent and incoherent production at fixed $x_{\mathbb{P}}=0.01$ are shown in Fig. 6. Here, we use the IPsat parametrization to describe the dipole-deuteron interaction. The effect of having different average density profiles for the gluon distribution results in coherent cross sections that deviate at $|t| \gtrsim 0.3 \mathrm{GeV}^{2}$. At smaller $|t|$, where one is sensitive to the structure at long distance scales, the spectra are identical.

Generally, the position of the first diffractive minimum corresponds to the size of the target $R$ as $t_{\mathrm{dip}} \sim 1 / R^{2}$. Here, the root mean square separations for the proton and neutron are similar in both wave functions, with the Hulthen wave function resulting in deuterons that are slightly larger by $\sim 3 \%$. So the difference in the observed spectra must be due to the 


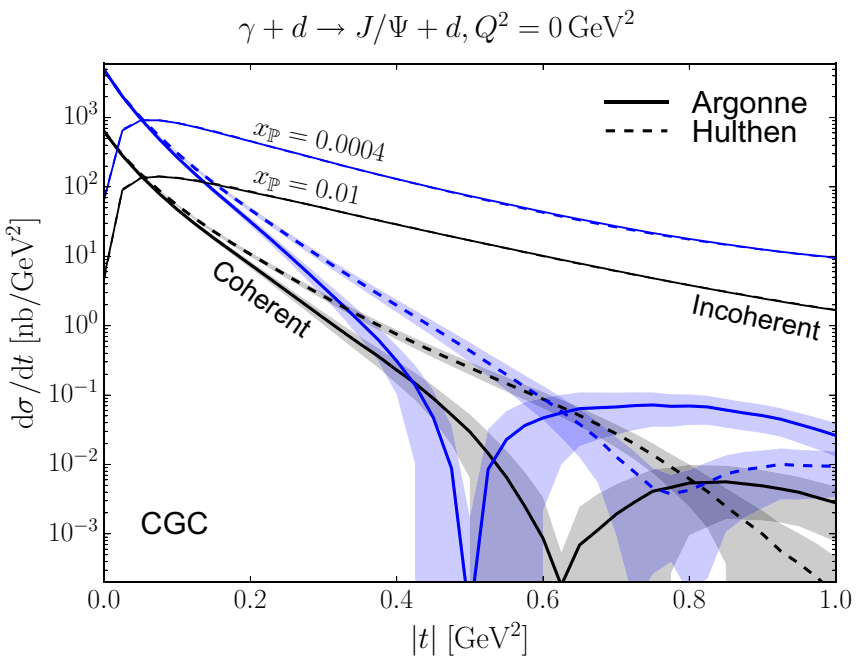

FIG. 7. Coherent and incoherent $J / \Psi$ production cross sections at $x_{\mathbb{P}}=0.01$ (lower black lines) and $x_{\mathbb{P}}=0.0004$ (upper blue lines) as a function of $t$, calculated from the CGC framework using two different wave functions for the deuteron at $x_{\mathbb{P}}=0.01$. Nucleon shape fluctuations are not included in the calculation.

different average shape (the $t$ spectra is the Fourier transform of the impact parameter profile, and the density profiles differ as seen in Fig. 1), not different average size. If the deuteron size is characterized by measuring the slope $B_{D}$ of the coherent cross section close to $t=0$ (parametrizing the spectra as $\sim e^{-B_{D}|t|}$ ), we find that the spectrum is slightly steeper when the Hulthen wave function is used $\left(B_{D} \approx 28 \mathrm{GeV}^{-2}\right.$ with Argonne potential wave function and $B_{D} \approx 29 \mathrm{GeV}^{-2}$ with Hulthen), consistent with the slightly larger rms size.

The incoherent cross sections are basically identical in the studied $|t|$ range, with the largest difference at $|t| \approx 0.1 \mathrm{GeV}^{2}$, where the result with the Argonne potential wave function is below the Hulthen result by $\approx 5 \%$. This can be understood because the short-range correlations effectively reduce overall density fluctuations by rejecting some of the configurations where the nucleons overlap in the transverse plane. However, the effect is numerically small and most likely not observable.

To study if the difference between the two wave functions for the deuteron is washed out by the small- $x$ evolution, we next show predictions for $J / \Psi$ photoproduction calculated from the CGC framework using both Hulthen and Argonne wave functions to describe the proton-neutron separation at $x_{\mathbb{P}}=0.01$. The energy evolution for each configuration is obtained by solving the JIMWLK equation. The resulting spectra at $x_{\mathbb{P}}=0.01$ and $x_{\mathbb{P}}=0.0004$ are shown in Fig. 7 . Here, we do not include nucleon shape fluctuations for simplicity. Similar to the case of the IPsat parametrization, the coherent cross sections obtained with different wave functions deviate above $|t| \gtrsim 0.2 \mathrm{GeV}^{2}$, and the position of the first diffractive minimum is at a smaller $|t|$ when the Argonne wave function is used. This difference is found to remain similar after the JIMWLK evolution down to $x_{\mathbb{P}}=0.0004$. This shows that the small- $x$ evolution retains the differences

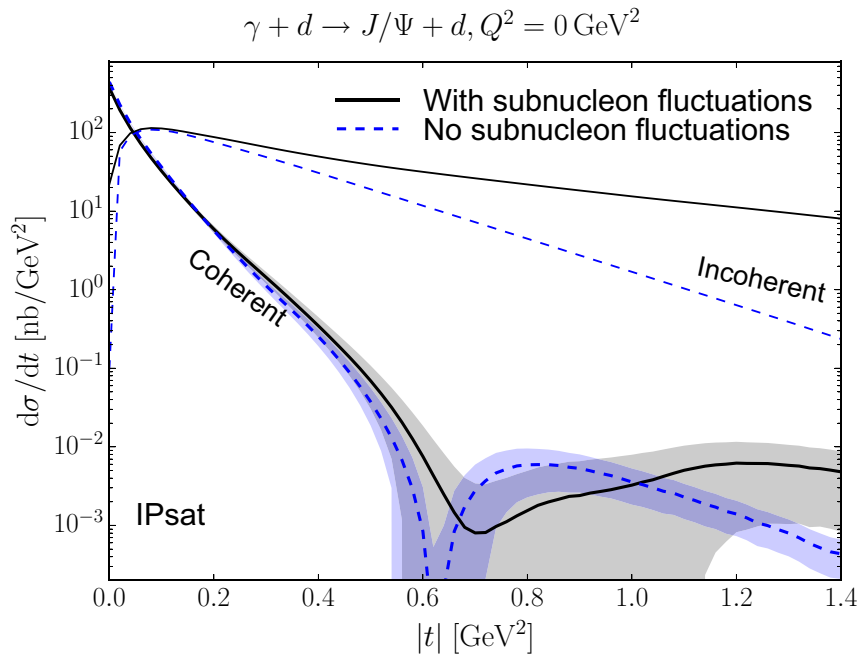

FIG. 8. Coherent and incoherent diffractive $J / \Psi$ photoproduction cross section in electron-deuteron collisions at $x_{\mathbb{P}}=0.01$, with (solid lines) and without (dashed lines) subnucleonic fluctuations.

between the deuteron wave functions defined at large $x$ within the $x$ range accessible with a future EIC.

\section{B. Deuteron shape and its small- $x$ evolution}

First we study the effect of nucleon shape fluctuations on diffractive $J / \Psi$ production off deuterons. In the following we only use the Argonne potential wave function to describe the proton-neutron separation in the deuteron. Our conclusions would be similar if the Hulthen wave function were used.

Using the IPsat model with and without nucleon shape fluctuations, we calculate the coherent and incoherent $J / \Psi$ photoproduction cross sections with deuteron targets at $x_{\mathbb{P}}=$ 0.01. The results are shown in Fig. 8. We find that the coherent cross sections are compatible within the numerical accuracy up to $|t| \gtrsim 1.2 \mathrm{GeV}^{2}$ with and without subnucleonic fluctuations (note that introducing the additional fluctuations approximately leaves the average shape unchanged). However, the incoherent cross sections start to deviate significantly at $|t| \approx 0.25 \mathrm{GeV}^{2}$, similar to the case of photon-heavy nucleus scattering analyzed in Ref. [10]. Note that at smaller $|t|$, where we are sensitive to fluctuations at longer length scales (scale of the proton and neutron separation, sampled from the deuteron wave function), there is basically no difference in the incoherent cross sections.

Next, we study the energy dependence of the cross sections. We calculate $J / \Psi$ production at different $x_{\mathbb{P}}$ values in the EIC energy range, and the results are shown in Fig. 9 for the IPsat model and in Fig. 10 for the CGC framework, where the JIMWLK evolution equation describes the structure evolution. The results are shown with nucleon shape fluctuations (solid lines) and without (dotted lines).

We find that the position of the first diffractive minimum moves to smaller $|t|$ as a result of the JIMWLK evolution in the color glass condensate calculation, which results in growing deuteron size as illustrated in Fig. 2. However, in the case of the IPsat parametrization where there is no geometry 


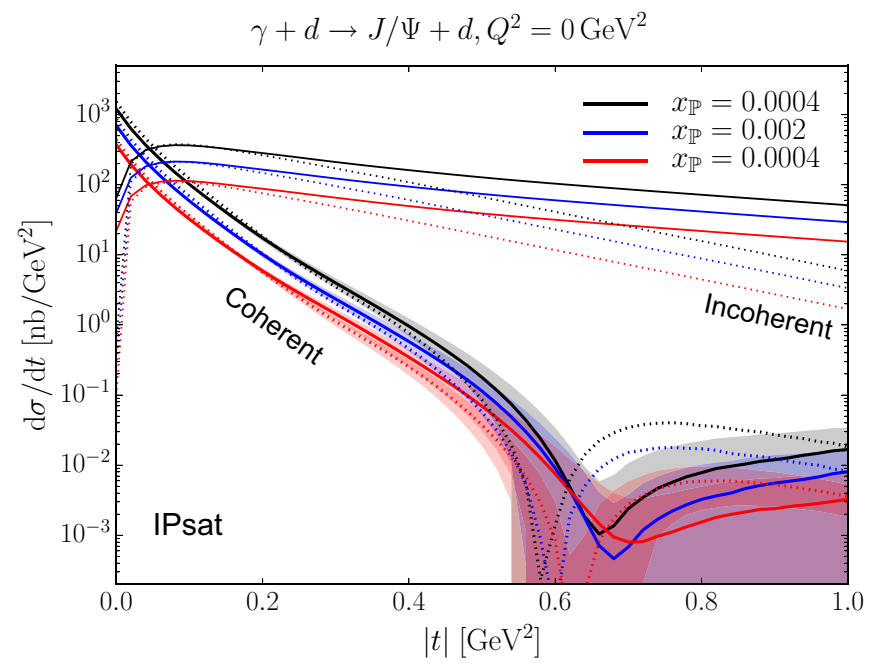

FIG. 9. Coherent and incoherent diffractive $J / \Psi$ photoproduction cross sections in photon-deuteron collisions at different $x_{\mathbb{P}}$ from the IPsat parametrization. Solid lines include nucleon shape fluctuations and dotted lines do not. For clarity, statistical uncertainty of the calculation is only shown for the case with fluctuating substructure, where errors are much larger.

evolution the dip location is approximately constant. Similarly, the coherent cross section at small $|t|$ gets steeper when the JIMWLK evolution is performed and remains constant in the IPsat calculation. This is demonstrated explicitly by parametrizing the coherent cross section as $d \sigma / d t \sim e^{-B_{D}|t|}$ and extracting the $|t|$ slope $B_{D}$ as a function of $x_{\mathbb{P}}$, shown in Fig. 11.

As shown in Ref. [9], the $|t|$ slope of the incoherent cross section $B$ is controlled by the size of the object that

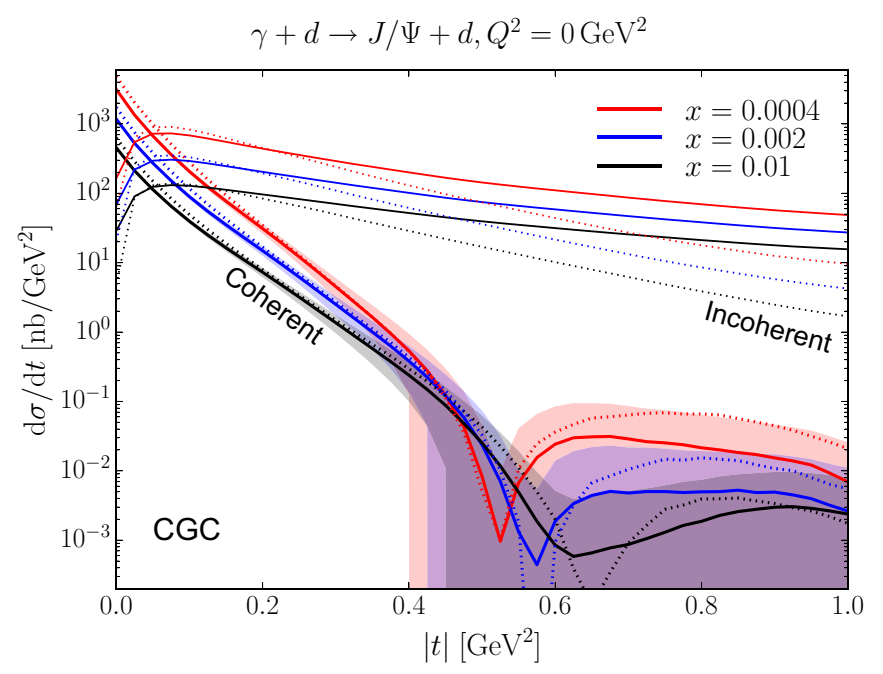

FIG. 10. Coherent and incoherent $J / \Psi$ photoproduction off deuterons calculated from the CGC framework at different $x_{\mathbb{P}}$. Solid lines include nucleon shape fluctuations and dotted lines do not. For clarity, statistical uncertainty of the calculation is only shown for the case with fluctuating substructure, where errors are much larger.

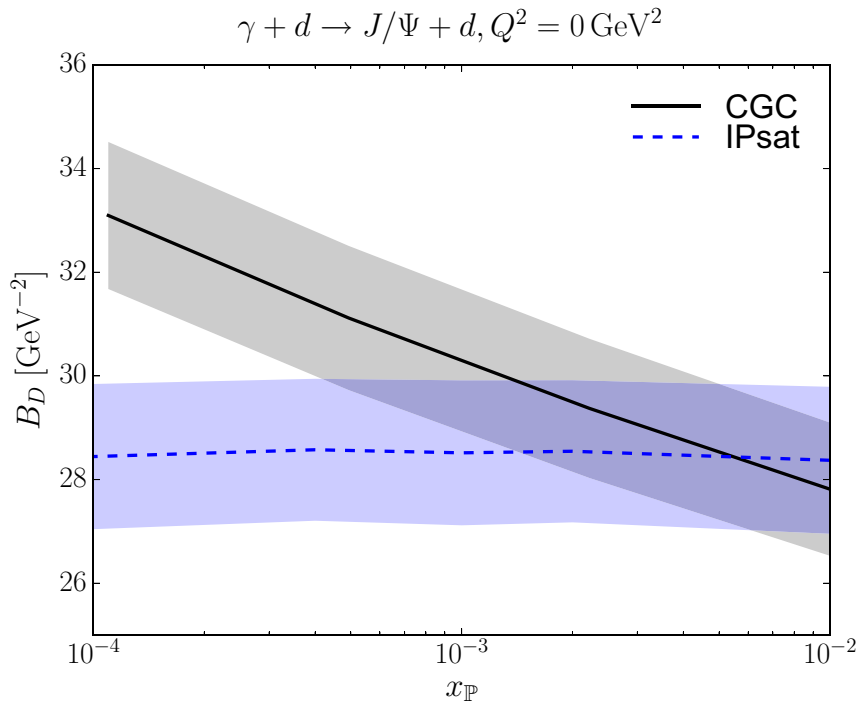

FIG. 11. Slope of the coherent cross section at small $|t|$ extracted from the calculation without nucleon shape fluctuations as a function of $x_{\mathbb{P}}$. The band shows the statistical uncertainty of the slope extraction.

is fluctuating. If the cross section is parametrized as $e^{-B|t|}$, at moderate $|t|$ the slope $B$ is controlled by the size of the nucleon, with $B \approx 4-5 \mathrm{GeV}^{-2}$ (note that the nucleon $\mathrm{rms}$ size is set by $B_{p}=4 \mathrm{GeV}^{-2}$ ). At large $|t|$, if the substructure fluctuations are included and the nucleons consist of hot spots, the slope approaches the hot spot size and $B \approx 1-2 \mathrm{GeV}^{-2}$ (recall that our hot spot size in the IPsat parametrization is $B_{q}=1 \mathrm{GeV}^{-2}$ ). The $|t|$ slopes extracted from the IPsat calculation of the incoherent cross section at two different Bjorken- $x$ values are shown in Fig. 12. As the size of the nucleons and hot spots does not depend on $x$, the extracted slopes are independent of $x$.

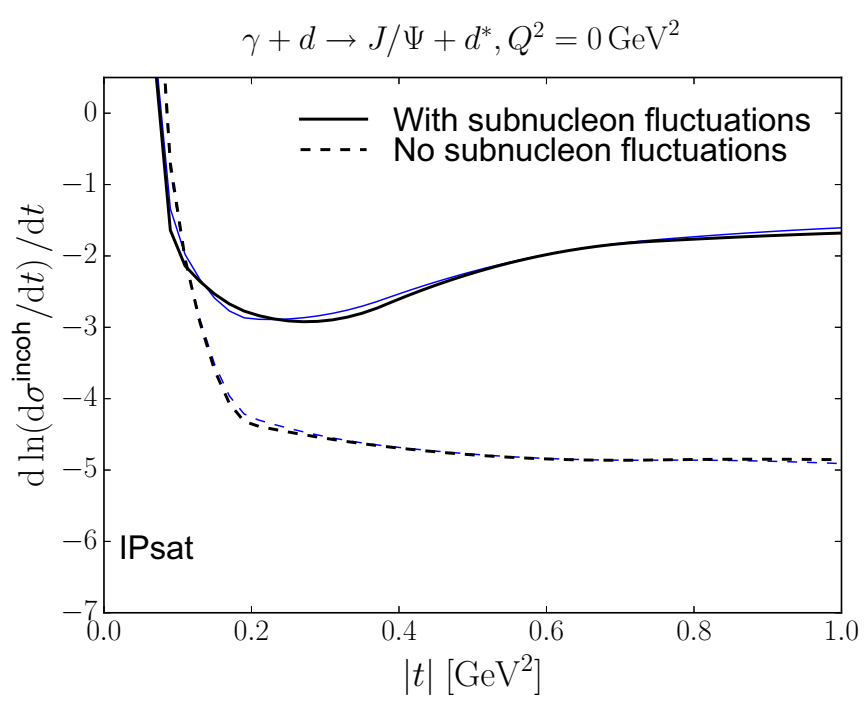

FIG. 12. Slope of the incoherent cross section in $\gamma+$ deuteron scattering from IPsat. Black lines are at $x_{\mathbb{P}}=0.01$ and blue lines at $x_{\mathbb{P}}=0.0004$. 


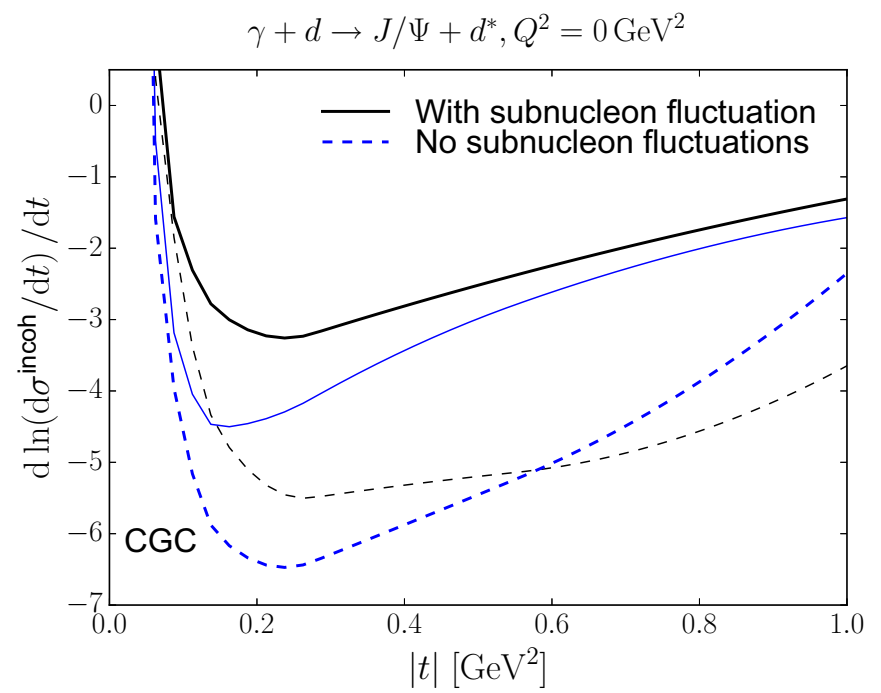

FIG. 13. Slope of the incoherent cross section in $\gamma+$ deuteron scattering from the CGC. Black lines are at $x_{\mathbb{P}}=0.01$ and blue lines at $x_{\mathbb{P}}=0.0004$.

In the CGC calculation, however, both nucleons and hot spots grow as a result of the small- $x$ evolution. The extracted slopes in this case are shown in Fig. 13. At moderate $|t| \approx$ $0.2 \mathrm{GeV}^{2}$ the result of the $x$ evolution is to make the spectra steeper, because of the growth of the system, both in the case with and without nucleon shape fluctuations. For any $x$, at large $|t|$ nucleon shape fluctuations start to dominate if included, similar to the IPsat calculation. Without nucleon shape fluctuations, the slopes are not constant at large $|t|$ in contrast to the IPsat calculation. This is due to the short-range color charge fluctuations in the target that contribute to the variance of the scattering amplitude. As a result of the small$x$ evolution, these fluctuations start to take place at shorter distance scales, and consequently the cross section falls more slowly as a function of $|t|$ after the evolution. Because of this, the slope as a function of $|t|$ for smaller $x$ crosses that for the initial $x$ at an intermediate $|t| \approx 0.6 \mathrm{GeV}^{2}$.

We note that, in the case of an infinite target, in the limit $|t| \gg Q_{s}^{2}$ and for small dipoles, the incoherent cross section approaches a power law, which dominates over the exponentially falling geometric component at large enough $|t|$. When the nucleon shape fluctuations are included, the short-range color charge fluctuations do not have a large effect as the results are very similar to the case of the IPsat calculation in the studied $|t|$ range.

\section{Evolution of the helium structure}

Similar to the case of deuterons, we study the effect of nucleon shape fluctuations in ${ }^{3} \mathrm{He}$ by calculating diffractive $J / \Psi$ photoproduction cross sections off helium-3 in the EIC kinematics, first using the IPsat parametrization to describe dipole-nucleon scattering. The results are shown in Fig. 14, where we again find that the effect of nucleon shape fluctuations changes the incoherent cross section at $|t| \gtrsim 0.2 \mathrm{GeV}^{2}$,

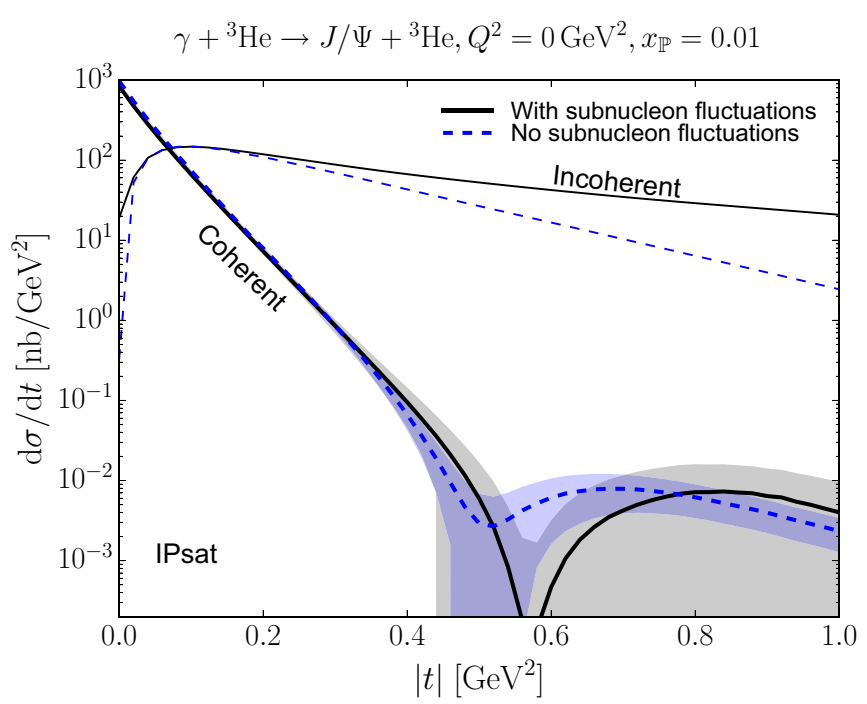

FIG. 14. Coherent and incoherent diffractive $J / \Psi$ photoproduction cross section in photon- ${ }^{3} \mathrm{He}$ collisions at $x_{\mathbb{P}}=0.01$ with (solid lines) and without (dashed lines) subnucleon fluctuations, calculated using the IPsat parametrization.

corresponding to the distance scale of the nucleon substructure used in our calculations.

To study the energy evolution of the helium structure illustrated in Fig. 3, we calculate coherent and incoherent $J / \Psi$ production cross sections at the initial condition and at smaller $x$ values accessible at the EIC. Similar to deuterons, the position of the first diffractive minimum moves to smaller $|t|$ as a result of the evolution, and the coherent spectra get steeper at small $|t|$. Incoherent cross sections in both cases are comparable up to $|t| \approx 0.2 \mathrm{GeV}^{2}$, after which the spectra

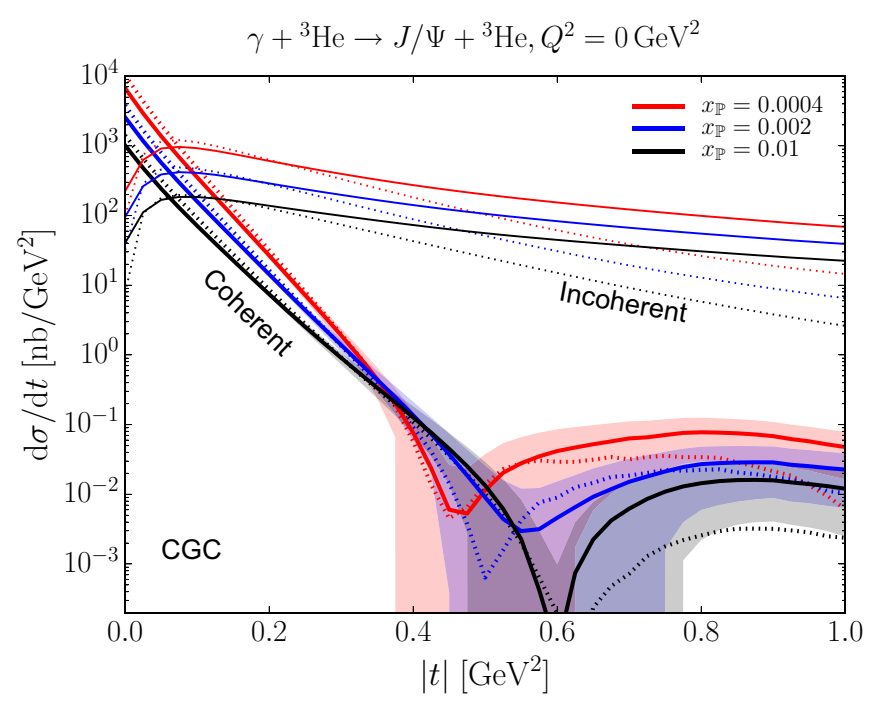

FIG. 15. Coherent and incoherent diffractive $J / \Psi$ photoproduction cross sections in photon- ${ }^{3} \mathrm{He}$ collisions at different $x_{\mathbb{P}}$. Solid lines include nucleon shape fluctuations and dotted lines do not. For clarity, statistical uncertainty of the calculation is only shown for the case with fluctuating substructure, where errors are much larger. 
are much more steeply falling in the case of no nucleon substructure fluctuations (see Fig. 15).

\section{CONCLUSIONS AND OUTLOOK}

We presented predictions for exclusive $J / \Psi$ photoproduction in the EIC kinematics with deuteron and helium-3 targets. We showed that employing two commonly used deuteron wave functions resulted in significantly different coherent vector meson spectra. This demonstrates the sensitivity for probing the transverse distribution of small- $x$ gluons at a future EIC. In particular, diffractive vector meson measurements at an EIC could reveal deviations in the spatial gluon distributions of light nuclei from the model assumptions in this work.

By solving the small- $x$ JIMWLK evolution equation, we predicted the energy dependence of the coherent and incoherent cross sections in the EIC energy range. The slope of the coherent $|t|$-dependent cross sections was found to become steeper due to the growth of the nucleus with decreasing $x$. The differences between the coherent cross sections computed with different wave functions at $x_{\mathbb{P}}=0.01$ remained similar down to $x_{\mathbb{P}}$ of a few times $10^{-4}$.

We showed that the incoherent cross section at $|t| \gtrsim$ $0.2 \mathrm{GeV}^{2}$ is sensitive to additional nucleon substructure fluctuations, which were previously constrained by HERA data. These results are similar to what was found for heavy nuclei [10]. The small- $x$ evolution did not significantly modify the $|t|$ value above which the nucleon substructure affects the incoherent cross section. However, the $|t|$ slope of the incoherent cross section also becomes steeper with decreasing $x$, which indicates the growth of the fluctuating constituents, namely, both nucleons $\left(|t| \approx 0.2 \mathrm{GeV}^{2}\right)$ and subnucleonic hot spots $\left(|t| \gtrsim 0.2 \mathrm{GeV}^{2}\right)$.

When not considering geometric subnucleon fluctuations, the slope of the incoherent vector meson spectra at $|t| \gtrsim$ $0.6 \mathrm{GeV}^{2}$ becomes flatter as a result of the evolution. This is because with increasing $Q_{s}$, color charge fluctuations appear on decreasing distance scales. This effect is absent in calculations without explicit color charge fluctuations, as shown in case of the IPsat model.

In addition to the fundamental information on the gluonic structure of light nuclei at small $x$ that exclusive vector meson production can provide, it also has applications for the phenomenology of high-energy nucleus-nucleus collisions. To interpret deuteron-gold and helium-gold measurements at RHIC, it is important to have precise knowledge of the small- $x$ geometry of light ions, which is an input for model calculations involving hydrodynamic simulations of QGP evolution (see Ref. [11] for a review and Refs. [13,58-61] for more recent developments).

Systematically going to heavier nuclei will be very interesting, as clustering of nucleons and other correlations, and their effect on the small- $x$ gluonic structure, could be probed by a future EIC as well (see also Refs. [62-64] for a discussion of systematics of saturation effects in heavier nuclei).

\section{ACKNOWLEDGMENTS}

We thank T. Haverinen, M. Mace, F. Salazar, and T. Ullrich for discussions. B.P.S. is supported under DOE Contract No. DE-SC0012704. H.M. is supported by the Academy of Finland, project 314764, and by the European Research Council, Grant No. ERC-2015-CoG-681707. H.M. wishes to thank the Nuclear Theory Group at BNL for hospitality during the final stages of this work. This research used resources of the National Energy Research Scientific Computing Center, which is supported by the Office of Science of the U.S. Department of Energy under Contract No. DE-AC02-05CH11231, CSC-IT Center for Science in Espoo, Finland, and the Finnish Grid and Cloud Infrastructure (persistent identifier urn:nbn:fi:research-infras-2016072533).
[1] F. D. Aaron et al. (H1 and ZEUS Collaborations), Combined measurement and QCD analysis of the inclusive $e^{ \pm} p$ scattering cross sections at HERA, J. High Energy Phys. 01 (2010) 109.

[2] H. Abramowicz et al. (H1 and ZEUS Collaborations), Combination of measurements of inclusive deep inelastic $e^{ \pm} p$ scattering cross sections and QCD analysis of HERA data, Eur. Phys. J. C 75, 580 (2015).

[3] H. Mäntysaari and B. Schenke, Evidence of Strong Proton Shape Fluctuations from Incoherent Diffraction, Phys. Rev. Lett. 117, 052301 (2016).

[4] H. Mäntysaari and B. Schenke, Revealing proton shape fluctuations with incoherent diffraction at high energy, Phys. Rev. D 94, 034042 (2016).

[5] H. I. Miettinen and J. Pumplin, Diffraction scattering and the parton structure of hadrons, Phys. Rev. D 18, 1696 (1978).

[6] L. Frankfurt, G. A. Miller, and M. Strikman, Evidence for Color Fluctuations in Hadrons from Coherent Nuclear Diffraction, Phys. Rev. Lett. 71, 2859 (1993).
[7] L. Frankfurt, M. Strikman, D. Treleani and C. Weiss, Evidence for Color Fluctuations in the Nucleon in High-Energy Scattering, Phys. Rev. Lett. 101, 202003 (2008).

[8] A. Caldwell and H. Kowalski, in Proceedings of the 13th International Conference on Elastic and Diffractive Scattering, Blois Workshop, Geneva, Switzerland, June-July 2009 (CERN, Geneva, 2010), pp. 190-192.

[9] T. Lappi and H. Mäntysaari, Incoherent diffractive J/ $\Psi$ production in high energy nuclear DIS, Phys. Rev. C 83, 065202 (2011).

[10] H. Mäntysaari and B. Schenke, Probing subnucleon scale fluctuations in ultraperipheral heavy ion collisions, Phys. Lett. B 772, 832 (2017).

[11] K. Dusling, W. Li, and B. Schenke, Novel collective phenomena in high-energy proton-proton and proton-nucleus collisions, Int. J. Mod. Phys. E 25, 1630002 (2016).

[12] B. Schenke and R. Venugopalan, Eccentric Protons? Sensitivity of Flow to System Size and Shape in $p+p, p+\mathrm{Pb}$ and $\mathrm{Pb}+\mathrm{Pb}$ Collisions, Phys. Rev. Lett. 113, 102301 (2014). 
[13] H. Mäntysaari, B. Schenke, C. Shen, and P. Tribedy, Imprints of fluctuating proton shapes on flow in proton-lead collisions at the LHC, Phys. Lett. B 772, 681 (2017).

[14] J. S. Moreland, J. E. Bernhard, and S. A. Bass, Estimating initial state and quark-gluon plasma medium properties using a hybrid model with nucleon substructure calibrated to $p-\mathrm{Pb}$ and $\mathrm{Pb}-\mathrm{Pb}$ collisions at $\sqrt{s_{\mathrm{NN}}}=5.02 \mathrm{TeV}$, arXiv:1808.02106.

[15] G. Antchev et al. (TOTEM Collaboration), Proton-proton elastic scattering at the LHC energy of $\sqrt{s}=7 \mathrm{TeV}$, Europhys. Lett. 95, 41001 (2011).

[16] J. L. Albacete and A. Soto-Ontoso, Hot spots and the hollowness of proton-proton interactions at high energies, Phys. Lett. B 770, 149 (2017).

[17] B. I. Abelev et al. (STAR Collaboration), Systematic measurements of identified particle spectra in $p p, d^{+} \mathrm{Au}$ and $\mathrm{Au}+\mathrm{Au}$ collisions from STAR, Phys. Rev. C 79, 034909 (2009).

[18] C. Aidala et al. (PHENIX Collaboration), Measurements of Multiparticle Correlations in $d+\mathrm{Au}$ Collisions at 200, 62.4, 39 , and $19.6 \mathrm{GeV}$ and $p+\mathrm{Au}$ Collisions at $200 \mathrm{GeV}$ and Implications for Collective Behavior, Phys. Rev. Lett. 120, 062302 (2018).

[19] C. Aidala et al. (PHENIX Collaboration), Creation of quarkgluon plasma droplets with three distinct geometries, Nat. Phys. 15, 214 (2019).

[20] A. Accardi et al., Electron ion collider: The next QCD frontier, Eur. Phys. J. A 52, 268 (2016).

[21] H. Kowalski and D. Teaney, An impact parameter dipole saturation model, Phys. Rev. D 68, 114005 (2003).

[22] J. Jalilian-Marian, A. Kovner, L. D. McLerran, and H. Weigert, The intrinsic glue distribution at very small $x$, Phys. Rev. D 55, 5414 (1997).

[23] J. Jalilian-Marian, A. Kovner, A. Leonidov, and H. Weigert, The BFKL equation from the Wilson renormalization group, Nucl. Phys. B 504, 415 (1997).

[24] J. Jalilian-Marian, A. Kovner, A. Leonidov, and H. Weigert, The Wilson renormalization group for low $x$ physics: Towards the high density regime, Phys. Rev. D 59, 014014 (1998).

[25] E. Iancu and L. D. McLerran, Saturation and universality in QCD at small $x$, Phys. Lett. B 510, 145 (2001).

[26] R. B. Wiringa, V. G. J. Stoks, and R. Schiavilla, An accurate nucleon-nucleon potential with charge independence breaking, Phys. Rev. C 51, 38 (1995).

[27] www.phy.anl.gov/theory/research/density2.

[28] M. L. Miller, K. Reygers, S. J. Sanders, and P. Steinberg, Glauber modeling in high energy nuclear collisions, Annu. Rev. Nucl. Part. Sci. 57, 205 (2007).

[29] L. B. Weinstein, E. Piasetzky, D. W. Higinbotham, J. Gomez, O. Hen, and R. Shneor, Short Range Correlations and the EMC Effect, Phys. Rev. Lett. 106, 052301 (2011).

[30] O. Hen, D. W. Higinbotham, G. A. Miller, E. Piasetzky, and L. B. Weinstein, The EMC effect and high momentum nucleons in nuclei, Int. J. Mod. Phys. E 22, 1330017 (2013).

[31] O. Hen, G. A. Miller, E. Piasetzky, and L. B. Weinstein, Nucleon-nucleon correlations, short-lived excitations, and the quarks within, Rev. Mod. Phys. 89, 045002 (2017).

[32] J. Carlson and R. Schiavilla, Structure and dynamics of few nucleon systems, Rev. Mod. Phys. 70, 743 (1998).

[33] J. L. Nagle, A. Adare, S. Beckman, T. Koblesky, J. Orjuela Koop, D. McGlinchey, P. Romatschke, J. Carlson, J. E. Lynn, and M. McCumber, Exploiting Intrinsic Triangular Geometry in Relativistic $\mathrm{He}^{3}+\mathrm{Au}$ Collisions to Disentangle Medium Properties, Phys. Rev. Lett. 113, 112301 (2014).

[34] C. Loizides, J. Nagle, and P. Steinberg, Improved version of the PHOBOS Glauber Monte Carlo, SoftwareX 1-2, 13 (2015).

[35] M. L. Good and W. D. Walker, Diffraction dissociation of beam particles, Phys. Rev. 120, 1857 (1960).

[36] H. Kowalski, L. Motyka, and G. Watt, Exclusive diffractive processes at HERA within the dipole picture, Phys. Rev. D 74, 074016 (2006).

[37] J. Bartels, K. Golec-Biernat, and K. Peters, On the dipole picture in the nonforward direction, Acta Phys. Pol. B 34, 3051 (2003).

[38] G. Chen, Y. Li, P. Maris, K. Tuchin, and J. P. Vary, Diffractive charmonium spectrum in high energy collisions in the basis light-front quantization approach, Phys. Lett. B 769, 477 (2017).

[39] T. Lappi and H. Mäntysaari, J/ $\Psi$ production in ultraperipheral $\mathrm{Pb}+\mathrm{Pb}$ and $p+\mathrm{Pb}$ collisions at energies available at the CERN Large Hadron Collider, Phys. Rev. C 87, 032201 (2013).

[40] A. G. Shuvaev, K. J. Golec-Biernat, A. D. Martin, and M. G. Ryskin, Off diagonal distributions fixed by diagonal partons at small $x$ and $\xi$, Phys. Rev. D 60, 014015 (1999).

[41] A. H. Rezaeian, M. Siddikov, M. Van de Klundert, and R. Venugopalan, Analysis of combined HERA data in the impactparameter dependent saturation model, Phys. Rev. D 87, 034002 (2013).

[42] H. Mäntysaari and P. Zurita, In depth analysis of the combined HERA data in the dipole models with and without saturation, Phys. Rev. D 98, 036002 (2018).

[43] C. Alexa et al. (H1 Collaboration), Elastic and protondissociative photoproduction of $\mathrm{J} / \Psi$ mesons at HERA, Eur. Phys. J. C 73, 2466 (2013).

[44] B. Schenke, P. Tribedy, and R. Venugopalan, Multiplicity distributions in $p+p, p+A$, and $A+A$ collisions from Yang-Mills dynamics, Phys. Rev. C 89, 024901 (2014).

[45] L. McLerran and P. Tribedy, Intrinsic fluctuations of the proton saturation momentum scale in high multiplicity $p+p$ collisions, Nucl. Phys. A 945, 216 (2016).

[46] A. Krasnitz and R. Venugopalan, Nonperturbative computation of gluon minijet production in nuclear collisions at very high energies, Nucl. Phys. B 557, 237 (1999).

[47] T. Lappi, Production of gluons in the classical field model for heavy ion collisions, Phys. Rev. C 67, 054903 (2003).

[48] B. Schenke, P. Tribedy, and R. Venugopalan, Fluctuating Glasma Initial Conditions and Flow in Heavy Ion Collisions, Phys. Rev. Lett. 108, 252301 (2012).

[49] L. D. McLerran and R. Venugopalan, Computing quark and gluon distribution functions for very large nuclei, Phys. Rev. D 49, 2233 (1994).

[50] T. Lappi, Wilson line correlator in the MV model: Relating the glasma to deep inelastic scattering, Eur. Phys. J. C 55, 285 (2008).

[51] H. Mäntysaari and B. Schenke, Confronting impact parameter dependent JIMWLK evolution with HERA data, Phys. Rev. D 98, 034013 (2018).

[52] J.-P. Blaizot, E. Iancu, and H. Weigert, Nonlinear gluon evolution in path integral form, Nucl. Phys. A 713, 441 (2003)

[53] T. Lappi and H. Mäntysaari, On the running coupling in the JIMWLK equation, Eur. Phys. J. C 73, 2307 (2013). 
[54] K. J. Golec-Biernat and A. M. Stasto, On solutions of the Balitsky-Kovchegov equation with impact parameter, Nucl. Phys. B 668, 345 (2003).

[55] S. Schlichting and B. Schenke, The shape of the proton at high energies, Phys. Lett. B 739, 313 (2014).

[56] A. Kovner and U. A. Wiedemann, Nonlinear QCD evolution: Saturation without unitarization, Phys. Rev. D 66, 051502 (2002).

[57] E. C. Aschenauer, S. Fazio, J. H. Lee, H. Mäntysaari, B. S. Page, B. Schenke, T. Ullrich, R. Venugopalan, and P. Zurita, The electron-ion collider: Assessing the energy dependence of key measurements, Rep. Prog. Phys. 82, 024301 (2019).

[58] P. Romatschke, Light-heavy ion collisions: A window into preequilibrium QCD dynamics? Eur. Phys. J. C 75, 305 (2015).

[59] C. Shen, J.-F. Paquet, G. S. Denicol, S. Jeon, and C. Gale, Collectivity and electromagnetic radiation in small systems, Phys. Rev. C 95, 014906 (2017).
[60] R. D. Weller and P. Romatschke, One fluid to rule them all: Viscous hydrodynamic description of event-by-event central $\mathrm{p}+\mathrm{p}, \mathrm{p}+\mathrm{Pb}$, and $\mathrm{Pb}+\mathrm{Pb}$ collisions at $\sqrt{s}=5.02 \mathrm{TeV}$, Phys. Lett. B 774, 351 (2017).

[61] B. Schenke, C. Shen, and P. Tribedy, Hybrid color glass condensate and hydrodynamic description of the Relativistic Heavy Ion Collider small system scan, arXiv:1908.06212.

[62] H. Kowalski, T. Lappi, and R. Venugopalan, Nuclear Enhancement of Universal Dynamics of High Parton Densities, Phys. Rev. Lett. 100, 022303 (2008).

[63] H. Kowalski, T. Lappi, C. Marquet, and R. Venugopalan, Nuclear enhancement and suppression of diffractive structure functions at high energies, Phys. Rev. C 78, 045201 (2008).

[64] H. Mäntysaari and R. Venugopalan, Systematics of strong nuclear amplification of gluon saturation from exclusive vector meson production in high energy electron-nucleus collisions, Phys. Lett. B 781, 664 (2018). 